\title{
Lo mejor del Congreso 2019 del American College of Cardiology
}

\author{
Dres. Sofía Noria1, Sebastián Lorenzo², Carlos Guamán³, \\ Yamel Ache4, María Victoria Ramos²
}

\begin{abstract}
Resumen
Como todos los años, en esta oportunidad se celebró el 68o Congreso del Colegio Americano de Cardiología (ACC, por su sigla en inglés) entre los días 16 y 18 de marzo en Nueva Orleans, Luisiana. Ambientado en la ciudad del Mardi Gras y el jazz, nuevamente convocó a la cardiología mundial para promover el conocimiento a través de la presentación de múltiples actividades científicas. Contó con la presencia de más de 16.000 asistentes y se recibieron 2.300 artículos, muchos de los cuales, sin duda, modificarán la práctica clínica actual. Cabe destacar, asimismo, la presentación de la Guía de Prevención Primaria de la Enfermedad Cardiovascular, donde resalta el casi total abandono del ácido acetilsalicílico en prevención primaria debido a la falta de beneficio neto.

Realizaremos un breve resumen de algunos de los principales trabajos científicos presentados:

- Antithrombotic Therapy after Acute Coronary Syndrome or PCI in Atrial Fibrillation - The AUGUSTUS Trial

- Transcatheter Aortic-Valve Replacement with a Balloon-Expandable Valve in Low-Risk Patients - The PARTNER 3 trial

- The Safety and Efficacy of Femoral Access in STEMI: The SAFARI-STEMI Trial

- One-Month Dual Antiplatelet Therapy Followed by Clopidogrel Monotherapy versus Standard 12-Month Dual Antiplatelet Therapy With Clopidogrel After Drug-Eluting Stent Implantation. STOP-DAPT 2 Trial

- Results of a Large-scale, App-based Study to Identify Atrial Fibrillation Using a Smartwatch: The Apple Heart Study

Palabras clave: CARDIOLOGÍA

CONGRESOS

RESÚMENES
\end{abstract}

\section{The best of the 2019 American College of Cardiology Congress}

\section{Summary}

As every year, on this occasion the 68th Congress of the American College of Cardiology was held from March 16 to 18 in New Orleans, Louisiana. Set in the city of Mardi Gras and jazz, it once again called on global cardiology to promote knowledge through the presentation of multiple scientific activities. It counted with the presence of more than 16.000 attendees and 2.300 articles were received, many of which will undoubtedly modify the current clinical practice. It is also worth mentioning the presentation of the Primary Prevention of Cardiovascular Disease Guideline, which highlights the almost total discontinuation of acetylsalicylic acid in primary prevention due to the lack of net benefit.

We will make a brief summary of some of the main scientific papers presented:

- Antithrombotic Therapy after Acute Coronary Syndrome or PCI in Atrial Fibrillation - The AUGUSTUS Trial

- Transcatheter Aortic-Valve Replacement with a Balloon-Expandable Valve in Low-Risk Patients - The PARTNER 3 trial

- The Safety and Efficacy of Femoral Access in STEMI: The SAFARI-STEMI Trial

- One-Month Dual Antiplatelet Therapy Followed by Clopidogrel Monotherapy versus Standard 12-Month Dual Antiplatelet Therapy With Clopidogrel After Drug-Eluting Stent Implantation. STOP-DAPT 2 Trial

- Results of a Large-scale, App-based Study to Identify Atrial Fibrillation Using a Smartwatch: The Apple Heart Study

Key words: $\quad$ CARDIOLOGY

CONGRESSES

ABSTRACTS

1. Editora adjunta de Revista Uruguaya de Cardiología.

2. Centro Cardiovascular Casa de Galicia. Montevideo, Uruguay.

3. Centro Cardiovascular Universitario. Hospital de Clínicas, Universidad de la República. Montevideo, Uruguay.

4. Centro Cardiológico Americano. Montevideo, Uruguay.

Correspondencia: Dra. Sofía Noria. Correo electrónico: sofianb278@gmail.com

Los autores declaran no tener conflictos de intereses.

Recibido Abr 26, 2019; aceptado May 7, 2019. 


\section{O melhor do Congresso do Colégio Americano de Cardiologia 2019}

\section{Resumo}

Como todos os anos, nesta ocasião, o 68ํㅡㄹ Congresso do Colégio Americano de Cardiologia (ACC após sua sigla em inglês) foi realizado de 16 a 18 de março em Nova Orleans, Louisiana. Situado na cidade do Mardi Gras ejazz, mais uma vez convidou a cardiologia do mondo para promover o conhecimento através da apresentação de múltiplas atividades científicas. Contou com a presença de mais de 16.000 participantes e 2.300 artigos recebidos, muitos dos quais sem dúvida modificarão a prática clínica atual. Também vale ressaltar a apresentação da Diretriz para a Prevenção Primária de Doenças Cardiovasculares, que destaca o quase total abandono do ácido acetilsalicílico na prevenção primária devido à falta de benefício líquido. Faremos um breve resumo de alguns dos principais trabalhos científicos apresentados:

- Antithrombotic Therapy after Acute Coronary Syndrome or PCI in Atrial Fibrillation - The AUGUSTUS Trial - Transcatheter Aortic-Valve Replacement with a Balloon-Expandable Valve in Low-Risk Patients - The PARTNER 3 trial - The Safety and Efficacy of Femoral Access in STEMI: The SAFARI-STEMI Trial

- One-Month Dual Antiplatelet Therapy Followed by Clopidogrel Monotherapy versus Standard 12-Month Dual Antiplatelet Therapy With Clopidogrel After Drug-Eluting Stent Implantation. STOP-DAPT 2 Trial

- Results of a Large-scale, App-based Study to Identify Atrial Fibrillation Using a Smartwatch: The Apple Heart Study

Palavras chave: CARDIOLOGIA

CONGRESSOS

RESUMOS

\section{Antithrombotic Therapy after Acute Coronary Syndrome or $\mathrm{PCI}$ in Atrial Fibrillation - The AUGUSTUS Trial}

En pacientes anticoagulados por fibrilación auricular (FA) que presentan un síndrome coronario agudo (SCA) o una intervención coronaria percutánea (ICP) existen dudas acerca de la terapia antitrombótica adecuada ${ }^{(1)}$.

Dada la escasez de datos en cuanto al uso de apixabán en pacientes con FA y doble antiagregación, con el fin de evaluar la eficacia y seguridad en este contexto se realizó el estudio AUGUSTUS. Fue un ensayo clínico abierto, aleatorizado y controlado, en el que se incluyeron 4.614 pacientes de 33 países, que tuvieran indicación de anticoagulación por FA y además presentaran SCA o ICP, requiriendo tratamiento con un inhibidor P2Y12 durante al menos seis meses ${ }^{(1,2)}$. Se excluyeron pacientes que tuvieran contraindicación para recibir doble terapia antiagregante y aquellos con necesidad de antagonistas de la vitamina $\mathrm{K}$ (AVK) por otras razones: prótesis valvular, estenosis mitral moderada/severa.

El diseño fue factorial $2 \times 2$ con una fase inicial abierta en la que se randomizaron los pacientes a recibir anticoagulación con $5 \mathrm{mg}$ de apixabán dos veces al día (2,5 mg dos veces al día en pacientes seleccionados) o AVK con INR objetivo entre 2 y 3 . Cada uno de estos grupos era posteriormente asignado en otra fase doble ciego a recibir aspirina o placebo por seis me$\operatorname{ses}^{(1,2)}$. El objetivo primario fue de seguridad: presencia de sangrado mayor (según la International Society on Thrombosis and Haemostasis) o sangrado menor clínicamente relevante, mientras que el objetivo secundario (de eficacia) incluyó un compuesto de muerte/hospitalización y muerte/eventos isquémi$\cos ^{(1)}$.
La población incluida fue de 70 años promedio, $30 \%$ sexo femenino, con $\mathrm{CHA}_{2} \mathrm{DS}_{2}$-VASc de $3,9 \pm 1,6$. El tratamiento con inhibidor P2Y12 fue realizado en su amplia mayoría (90\%) con clopidogrel. En relación con el tipo de evento, aproximadamente $60 \%$ presentó SCA (60\% se realizó ICP), mientras que la ICP electiva fue realizada en $40 \%$.

Los resultados fueron presentados por el Dr. Renato Lopes (MD, MHS, Duke University, North Carolina) y publicados en la revista New England Journal of Medicine. El objetivo primario fue de $10,5 \%$ en los pacientes que recibieron apixabán versus $14,7 \%$ en el grupo AVK (HR 0,69; IC95\%, 0,58-0,81; p<0,001 tanto para no inferioridad como superioridad) y $16,1 \%$ en el grupo aspirina, comparado con $9 \%$ de los que recibieron placebo (HR 1,89; IC95\%, 1,59-2,24; p<0,001)(1). Cuando se compararon las cuatro ramas de tratamiento se observó que la combinación AVK + aspirina fue la que presentó mayor sangrado $(18,7 \%)$, mientras que la de menor sangrado fue apixabán + placebo $(7,3 \%)$.

Con respecto al objetivo secundario, los pacientes en el grupo de apixabán tuvieron una menor incidencia del compuesto muerte/hospitalización que aquellos bajo tratamiento con AVK (23,5\% vs 27,4\%; HR 0,83; IC95\%, 0,74-0,93; $\mathrm{p}=0,002$ ) mientras que no se observaron diferencias significativas en el caso de aspirina contra placebo. La comparación de AVK versus apixabán y aspirina versus placebo mostró similar incidencia de eventos isquémicos ${ }^{(1)}$.

Los autores concluyeron que en los pacientes con FA y SCA o ICP recientes tratados con un inhibidor P2Y12, el régimen antitrombótico con apixa- 
bán, sin aspirina, resultó en menos sangrados y hospitalizaciones sin diferencias significativas en la incidencia de eventos isquémicos en comparación con aquellos que incluían AVK, aspirina o ambos ${ }^{(1)}$.

Consultado al respecto, el Dr. Lopes dijo: "Debido a la preocupación sobre el sangrado mayor, han surgido preguntas sobre el adecuado tratamiento de pacientes con FA y SCA y/o sometidos a ICP. Los resultados de este estudio aportan información adicional para los médicos que tratan a estos pacientes de alto riesgo"(3).

\section{Transcatheter Aortic-Valve Replacement with a Balloon-Expandable Valve in Low-Risk Patients - The PARTNER 3 Trial}

El implante valvular aórtico transcatéter (TAVI) se ha posicionado como una alternativa a la sustitución valvular aórtica quirúrgica (SVAq) en pacientes con estenosis aórtica severa (EAoS) de moderado, alto y extremo riesgo quirúrgico(4). El TAVI fue superior -o no inferior- a la SVAq en estudios randomizados y grandes registros con válvulas tanto autoexpandibles como balón-expandibles $^{(4)}$. En lo que respecta a pacientes de bajo riesgo quirúrgico, en 2015 fue publicado el estudio NOTION, en el que se randomizaron pacientes con EAoS a TAVI (139 pacientes) o SVAq (135 pacientes) sin enfermedad arterial coronaria que requiriera intervención ${ }^{(5)}$. De estos, $82 \%$ presentaba riesgo quirúrgico bajo (evaluado mediante un score predictor de mortalidad de la Sociedad de Cirujanos Torácicos [STS-PROM] menor a 4\%). En el seguimiento a cinco años no hubo diferencias significativas en el objetivo final primario de muerte por cualquier causa, accidente cerebrovascular (ACV) e infarto $(39,2 \%$ para TAVI vs $35,8 \%$ para cirugía, $\mathrm{p}=0,78)^{(6)}$.

Pese a este resultado, las guías aún recomiendan la SVAq en pacientes con riesgo quirúrgico bajo $^{(4)}$. Con el objetivo de ampliar la evidencia del uso de TAVI en este grupo fue realizado el estudio PARTNER 3, presentado el 17 de marzo por el Dr. Martin Leon (MD, Presbyterian Hospital, New York) y simultáneamente publicado en la revista The New England Journal of Medicine ${ }^{(7)}$.

Este estudio multicéntrico randomizó, con relación 1:1, 1.000 pacientes con EAoS de bajo riesgo quirúrgico a TAVI utilizando válvula SAPIEN 3 (balón-expandible) por acceso femoral versus SVAq con válvula biológica ${ }^{(7)}$. El bajo riesgo clínico fue definido con un STS-PROM $<4 \%$. Se excluyeron pacientes con fragilidad clínica, válvula aórtica bicúspide o unicúspide $\mathrm{u}$ otras alteraciones anatómicas que incrementaran el riesgo de complicaciones del procedimiento (insuficiencia aórtica severa). De los 1.000 pacientes randomizados, 50 no fueron incluidos en el análisis, la mayoría por rechazar el procedimiento o preferir otro centro no perteneciente al estudio. Se realizó otro procedimiento en $7,9 \%$ de los pacientes sometidos a TAVI y en $26,4 \%$ de los sometidos a $\operatorname{SVAq}^{(7)}$.

La edad media fue 73 años (69\% hombres), y el STS score promedio fue de $1,9 \%(7,8)$. El objetivo primario -compuesto de muerte por todas las causas, ACV o rehospitalización al año- fue 8,5\% en el grupo TAVI vs $15,1 \%$ en el grupo SVAq, (HR 0,54; IC95\%, 0,37-0,79; p=0,001), demostrándose la superioridad de TAVI ${ }^{(7,8)}$. Del análisis de los componentes del objetivo primario por separado hubo una tendencia a favor de TAVI en todos ellos ${ }^{(7)}$.

Dentro de los objetivos secundarios los resultados a 30 días mostraron menores tasas de ACV $(0,6 \%$ vs $2,4 \% ; \mathrm{p}=0,02)$, ACV y muerte $(1,0 \%$ vs $3,3 \% ; \mathrm{p}=0,01)$, y de nuevo inicio de FA (5,0\% vs $39,5 \% ; \mathrm{p}<0,001)$ en el grupo TAVI ${ }^{(7,8)}$. A su vez, la tasa anual de muerte o ACV discapacitante fue $1 \%$ en el grupo TAVI vs $2,9 \%$ en el grupo quirúrgico (HR 0,34; IC95\%, 0,12-0,97)(7).

El implante de TAVI también tuvo menor índice de hospitalización que la cirugía (3 días versus 7 días, $\mathrm{p}<0,001)^{(7)}$ y mejoría más rápida en la clase funcional de la NYHA, la distancia en el test de caminata de 6 minutos, y el score del Kansas City Cardiomyopathy Questionnaire sobre calidad de vida $^{(8)}$.

Con respecto a los objetivos de seguridad a 30 días (complicaciones vasculares mayores, implante de marcapaso permanente, leak paravalvular aórtico moderado o severo u obstrucción coronaria), no hubo diferencias significativas entre los grupos. Sin embargo, la tasa de sangrado mayor o que pusiera en peligro la vida en el grupo TAVI fue 3,6\%, comparado con $24,5 \%$ en el grupo quirúrgico (HR 0,12 ; IC95\%, 0,07-0,21)(7).

Es de destacar que al año la incidencia de bloqueo completo de rama izquierda fue $32,7 \%$ en el grupo TAVI y 8,0\% en el grupo quirúrgico (HR 3,43 ; IC95\%, 2,32-5,08) y que la tasa de leak paravalvular leve también favoreció a la cirugía $(29,4 \%$ vs $2,1 \%)^{(7,8)}$.

Como principal limitante del estudio los autores mencionaron que solo refleja los resultados a un año y que no evalúa el problema del deterioro es- 
tructural de la válvula a largo plazo. "Las conclusiones definitivas sobre las ventajas y desventajas de la TAVI comparada con la cirugía dependen del seguimiento a largo plazo", mencionaron al final de la presentación ${ }^{(8)}$

Para recalcar la relevancia del estudio luego de la presentación, el Dr. Braunwald mencionó: "Este es un momento histórico y todos los aquí presentes deberíamos reconocer esto como tal. (...) Le contaremos a nuestros nietos que estuvimos ahí en el momento en que este avance increíble en el cuidado de los pacientes con estenosis aórtica fue presenta$d o "(9)$.

The Safety and Efficacy of Femoral Access versus Radial for Primary Percutaneous Coronary Intervention in ST-Elevation Myocardial Infarction: The SAFARI-STEMI Trial

La intervención cutánea percutánea (ICP) es el tratamiento de elección en el infarto agudo de miocardio con elevación del segmento ST (IAMCEST). Las últimas guías establecen el acceso radial como la técnica estándar para la angiografía coronaria y la ICP, con evidencia nivel I clase A, basados en los resultados del estudio MATRIX, donde el acceso radial se asoció a menor riesgo de hemorragia en el lugar de punción, complicaciones vasculares y necesidad de transfusión, así como a menor mortalidad, reforzando los resultados de los estudios RIVAL y RIFLE-STEACS(10,11).

Considerando que muchos centros intervencionistas en Estados Unidos y Canadá prefieren el acceso femoral (pese a la evidencia favorable sobre el acceso radial), fue diseñado el estudio SAFARISTEMI con el objetivo de comparar ambos accesos utilizando nueva farmacoterapia y tecnología (no incluidas previamente) en pacientes sometidos a una ICP por IAMCEST ${ }^{(12)}$.

SAFARI-STEMI fue un estudio randomizado, abierto, de asignación paralela y evaluación ciega, con un seguimiento de 30 días. El objetivo primario fue mortalidad por todas las causas y dentro de los objetivos secundarios se consideró el ACV, reinfarto, trombosis del stent o sangrado. Se incluyeron 2.292 pacientes con IAMCEST con inicio de síntomas $<12$ horas, derivados para ICP (edad media 62 años, índice de masa corporal $28,2,22 \%$ mujeres, $17 \%$ diabéticos) de cinco centros médicos de Canadá. Fueron randomizados 1.136 pacientes para acceso radial y 1.156 para acceso femoral. Todos recibieron $160 \mathrm{mg}$ de aspirina, carga de inhibidor P2Y12 y $60 \mathrm{UI} / \mathrm{kg}$ (máximo 4.000 UI) de heparina no fraccionada. Se excluyeron pacientes que recibieron fibrinolíticos, anticoagulantes orales y aquellos con cirugía de revascularización miocárdica previa(12-14)

Se destaca que en ambos grupos la mayoría de los pacientes recibieron tratamiento con bivalirudina durante el procedimiento y ticagrelor posteriormente; los dispositivos de cierre vascular fueron empleados en $68,2 \%$ dentro del grupo femoral y en $5,5 \%$ en el grupo radial. La tasa de crossover fue de $8,1 \%$ en el acceso radial y de $2,3 \%$ en el acceso femoral. No se dio a conocer cuántos accesos se realizaron bajo guía de ultrasonido y con kit de micropunción ${ }^{(12,13,15)}$.

Inicialmente los investigadores planearon incluir 4.884 pacientes, pero el estudio fue terminado de forma temprana debido a futilidad, sin diferencias en el objetivo primario (mortalidad por todas las causas a los 30 días: $1,5 \%$ en el grupo radial vs $1,3 \%$ en el grupo femoral, $p=0,69)$. Tampoco se encontraron diferencias en los objetivos secundarios, a saber: reinfarto (1,8\% radial vs $1,6 \%$ femoral, $\mathrm{p}=0,83), \operatorname{ACV}(1,0 \%$ vs $0,4 \% \mathrm{p}=0,12)$ y sangrado mayor por definición tanto TIMI como BARC (1,1\% vs $1,3 \% \mathrm{p}=0,74)$. Concluyen que en pacientes con IAMCEST sometidos a ICP primaria, el acceso radial no es superior al acceso femoral, y que operadores adecuadamente entrenados deberían ser capaces de obtener resultados similares usando cualquiera de estos abordajes ${ }^{(12-15)}$.

Debido a que en algunas ocasiones es necesario cambiar el sitio de acceso durante el procedimiento, el presentador de este trabajo, Michel Le May (MD, FACC, University of Otawa Heart Institute, Canadá) expresó: "Creo que será importante que los programas de capacitación médica enfaticen la necesidad de ser competentes tanto en el acceso radial como en el femoral, es posible volverse poco entrenado al realizar uno de estos, y un énfasis constante en uno sobre el otro puede llevar a un aumento de las complicaciones"(14).

Claire Duvernoy (Michigan University), quien se formó como operadora transfemoral, calificó los resultados como "tranquilizadores". Ella opta por el abordaje radial en pacientes electivos, pero cuando el momento es crítico -como los casos de IAMCESTsu primer instinto es utilizar el acceso femoral. Mientras que Sunil Rao (Duke University), defensor del acceso radial, dijo: "Si bien este ensayo de poca po- 
tencia no mostró diferencias entre radial y femoral, no está claro si los resultados realmente buenos observados con acceso femoral se pueden lograr en la práctica clínica”. Recalcó, además, que la interrupción temprana del estudio hace que sea imposible obtener conclusiones firmes. Duvernoy mencionó a los medios que el uso de bivalirudina y dispositivos de cierre no son la práctica estándar en su hospital, por lo que extrapolar los resultados será un reto ${ }^{(16)}$.
Durante la presentación, Le May mostró datos de un metaanálisis actualizado que incluye los resultados de SAFARI-STEMI: se observó menor riesgo de mortalidad entre los pacientes con IAMCEST tratados por acceso radial, pero siendo el beneficio relativo próximo a la unidad (RR 0,78 ; IC95\% 0,61-0,99)(16).

\section{One-Month Dual Antiplatelet Therapy Followed by Clopidogrel Monotherapy vs.} Standard 12-Month Dual Antiplatelet Therapy with Clopidogrel After Drug-Eluting Stent Implantation. STOP-DAPT 2 Trial

La terapia antiagregante luego del intervencionismo cardiovascular es una temática que ocupa a la investigación actual, entre otros motivos porque los stents coronarios de última generación asocian un riesgo menor de trombosis a lo reportado con stents previos. Asimismo, el riesgo de sangrado vinculado a la doble antiagregación (DAPT) aumenta indefectiblemente con la duración del tratamiento y su mortalidad es comparable a la de un nuevo infarto de miocardio (IM). El estudio STOP-DAPT $2^{(17)}$ fue presentado el $18 \mathrm{de}$ marzo de 2019 por el Dr. Hirotoshi Watanabe (MD, Kyoto University, Japan), pero la publicación de sus resultados permanece aún pendiente. Se trata de un estudio aleatorizado y multicéntrico realizado en 90 centros japoneses, cuyo objetivo principal fue demostrar la seguridad y eficacia de la terapia experimental de DAPT por un mes seguida de monoterapia con clopidogrel en comparación con el DAPT estándar de 12 meses con aspirina y clopidogrel, luego del implante de un stent liberador de everolimus de última generación.

Los resultados presentados corresponden al análisis primario de no inferioridad a un año. El objetivo final primario fue un compuesto de muerte cardiovascular, IM, trombosis definitiva del stent, ACV y sangrado mayor o menor según el score Thrombolysis In Myocardial Infarction (TIMI). El objetivo final secundario incluyó el análisis independiente de los eventos isquémicos y hemorrágicos mencionados. Se excluyeron pacientes anticoagulados y con antecedente de hemorragia intracraneal. De los 6.504 pacientes que cumplían criterios de inclusión fueron randomizados 3.045 a una de las dos ramas de tratamiento, de los cuales 36 abandonaron el estudio, por lo que el análisis final incluyó un total de 3.009 pacientes: 1.500 recibieron DAPT por un mes y 1.509 DAPT durante 12 meses. La media de edad fue de 68 años y solo hubo $21 \%$ de población femenina. El $39 \%$ de los pacientes incluidos eran diabéticos y $62 \%$ tenía cardiopatía isquémica estable. En $83 \%$ se utilizó abordaje radial y $97 \%$ de las angioplastias fueron optimizadas por técnicas de imagen intravascular. Más del 90\% de los pacientes tenían un riesgo de trombosis y hemorragia intermedio o bajo, valorado por CREDO-Kyoto thrombotic risk score y CREDO-Kyoto bleeding risk score.

Los autores encontraron un beneficio neto de la DAPT durante un mes frente a la DAPT estándar, con un riesgo del evento primario de $2,4 \%$ vs $3,7 \%$ (valor p de no inferioridad $<0,001$ y de superioridad de 0,04). En el análisis independiente de eventos isquémicos y hemorrágicos se observó que la diferencia significativa del resultado combinado radica principalmente en la reducción de eventos hemorrágicos. Sin embargo, la no inferioridad de DAPT durante un mes persistió en el análisis al año del punto final secundario de eventos isquémicos $(2,0 \%$ versus $2,5 \%$, p de no inferiori$\mathrm{dad}=0,005$ ). El sangrado TIMI mayor y menor fue significativamente menor en el grupo de DAPT acortado $(0,4 \%$ frente a $1,5 \%$, valor p de no inferiori$\mathrm{dad}=0,002$ y valor $\mathrm{p}$ de superioridad $=0,004)$. " $E n$ conclusión, señoras y señores, el DAPT de un mes, seguido de la monoterapia con clopidogrel, brindó un beneficio clínico neto para los eventos de isquemia y sangrado(...) después del implante de un stent liberador de everolimus de última generación", dijo Watanabe. "El beneficio fue impulsado por una reducción significativa en el sangrado sin un aumento en los eventos isquémicos"(18).

La principal limitación de este trabajo radica en que la población estudiada tenía un riesgo isquémico bajo o intermedio, lo que imposibilita extrapolar resultados a pacientes con mayor riesgo. Por otra parte, la optimización de la intervención coronaria mediante imagen intravascular reduce el riesgo de trombosis del stent, pero se trata de 
una estrategia de uso restringido en nuestro medio. Asimismo, los pacientes japoneses tienen menor riesgo isquémico que la población europea y americana, aspecto resaltado durante la presentación ${ }^{(18,19)}$. Para conocer más extensamente los re- sultados de este trabajo esperamos con ansias su publicación.

\section{Results of a Large-scale, App-based Study to Identify Atrial Fibrillation Using a Smartwatch: The Apple Heart Study}

Los datos preliminares de este estudio ${ }^{(20)}$, presentados por el Dr. Mintu Turakhia (MD MAS, Standford University), fueron de los más esperados del Congreso. Frente a la popularidad del Apple Watch y otros dispositivos portátiles con aplicaciones disenadas para detectar el ritmo cardíaco, surge la posibilidad de identificar la presencia de arritmias comunes, especialmente la FA. Para ello, se diseñó un novedoso estudio, prospectivo y de brazo único que incluyó 419.297 participantes de Estados Unidos desde noviembre de 2017 a julio de 2018. Fueron criterios de inclusión tener un $i$ Phone 5 o versiones posteriores, Apple Watch serie 1 o posteriores y edad $\geq 22$ años. Se excluyeron pacientes con historia de FA, flutter auricular o terapia anticoagulante en el momento del reclutamiento.

El objetivo primario consistió en medir el porcentaje de participantes con pulso irregular detectados por el Apple Watch que fueron diagnosticados como FA mediante un parche que registra trazado de electrocardiograma (ECG). Como objetivos secundarios se planteó caracterizar la concordancia de pulso irregular por Apple Watch con ECG ambulatorio registrado simultáneamente y estimar la tasa de contacto con profesionales de la salud tras la detección de pulso irregular.

Aquellos individuos interesados que descargaron la aplicación y cumplían los criterios de inclusión fueron invitados a participar en el estudio. El algoritmo de identificación de pulso irregular usó ondas de fotopletismografía para crear un tacograma (frecuencia cardíaca en función del tiempo). Si un tacograma cumplía los criterios de irregularidad, se realizaba una detección prospectiva para reconfirmar los hallazgos. En caso de obtenerse cinco latidos irregulares consecutivos, se notificaba al participante, a través de la aplicación, la presencia de pulso irregular ofreciendo mediante telemedicina el contacto con un profesional y se le indicaba concurrir a un centro de salud o colocarse el parche con capacidad de registrar el ECG para confirmar la presencia de FA (monitoreo de al menos siete días), en cuyo caso era programada una segunda visita virtual.
De la cohorte global, $42 \%$ fueron mujeres (177.087), con franco predominio de participantes jóvenes $(52 \%$ -219.179- entre 22 y 39 años) de raza blanca $(68 \%$ -286.190-). Recibieron notificación de la aplicación 2.161 participantes $(0,52 \%)$, siendo en este caso $21 \%$ las mujeres (461) con predominio de $\geq 65$ años ( $36 \%$ -775-). Realizaron consulta virtual 945 participantes (44\%) y $30 \%$ fue enviado a urgencia por sintomatología asociada. $\mathrm{Al} 70 \%$ restante se le colocó registro de ECG con parche, pero solo se devolvieron 450 que fueron incluidos en el análisis (23\% mujeres, $40 \% \geq 65$ años). $\mathrm{El} \mathrm{CHA}_{2} \mathrm{DS}_{2} \mathrm{VASc} \geq 2$ fue de $13 \%$ en la cohorte global (55.277 participantes), aumentando francamente a $33 \%$ en los que recibieron notificación de ritmo irregular y en el grupo parche de ECG (38\%). Se observó el mismo comportamiento de los factores de riesgo cardiovascular individuales como obesidad, hipertensión arterial y diabetes mellitus.

En los resultados a ocho meses de monitoreo se evidenció un porcentaje bajo de notificación de pulso irregular de 0,52\% (2.161/419.297 participantes) existiendo una clara relación con la edad (tasa de notificación $3,2 \%$ en $\geq 65$ años, 775/24.626 participantes). Cuando se analizaron los ECG de los individuos con notificación de pulso irregular por la aplicación, solo en 34\% (153/450) se confirmó la presencia de FA, lo que implica que en $66 \%$ hubo "alarmas falsas”. Los valores predictivos positivos para la cohorte total de identificación de FA en ECG parche comparados con el tacograma y las notificaciones de pulso irregular registrados simultáneamente fueron de 0,71 y 0,84 , respectivamente. En cuanto a la duración de la FA (considerada por el dispositivo cuando fue $\geq 30$ segundos), $89 \%$ de los episodios duraron $\geq 1$ hora con $25,5 \% \geq 24$ horas. Otro aspecto a destacar fue que de los pacientes notificados de pulso irregular que completaron los 90 días de seguimiento (1.376/2.161, 64\%), 57\% (787/1.376 participantes) contactaron a un profesional de la salud por fuera de la aplicación y en estos casos se tomaron diversas conductas, como inicio de nueva medicación (28\%), referencia a un especialista (33\%) y estudios adicionales (36\%). Los eventos adversos informados fueron muy bajos (1.038/419.297), en su 
amplia mayoría no relacionados a la aplicación (1.022/1.038)

Como limitaciones se destacaron: abandono mayor del anticipado luego de la notificación (64\%) y que fueron entregados menos ECG parche de lo planeado, lo que disminuye la precisión y el diseño virtual del estudio, donde los datos son autoinformados por los participantes ${ }^{(21)}$. Tampoco se alcanzó el objetivo de inscripción de 500.000 participantes con $75.000 \geq 65$ años ${ }^{(22)}$.

A propósito del hecho de que solo se identificó en $34 \%$, el Dr. Turakhia expresó: "No significa que el $66 \%$ no tenía FA. Simplemente significa que la FA puede no haber estado allí en ese momento"(22). Con respecto a la novedosa experiencia virtual, resaltó: "Este estudio mejora nuestra comprensión de cómo funciona la aplicación y tecnología portátil en el mundo real y qué tan bien la tecnología puede detectar largos períodos de FA. Las notificaciones de irregularidades en el ritmo cardiaco fueron bajas, un hallazgo importante debido a las preocupaciones sobre la notificación excesiva, y pudimos ver qué sucedió después de que los participantes recibieran una notificación"(23).

Este estudio virtual es el primer paso para crear las bases de futuros estudios que busquen aprovechar las tecnologías portátiles como una forma de apoyar la salud cardiovascular. "Realmente representa un cambio de paradigma en la forma en que se pueden llevar a cabo los estudios clínicos", dijo Turakhia.

Sofía Noria, https://orcid.org/0000-0003-0681-9706 Sebastián Lorenzo, https://orcid.org/000-0003-2827-601X Carlos Guamán, https://orcid.org/0000-0002-1065-1988 Yamel Ache, https://orcid.org/0000-0001-9956-4081 María Victoria Ramos, https://orcid.org/0000-0002-6349-2781

\section{Bibliografía}

1. Lopes RD, Heizer G, Aronson R, Vora AN, Massaro T, Mehran R, et al. Antithrombotic Therapy after Acute Coronary Syndrome or PCI in Atrial Fibrillation. N Eng J Med. 2019;380(16):1509-24. doi: 10.1056/NEJMoa1817083

2. Ortiz Cortés C. Estudio AUGUSTUS. ACC 2019: Apixabán en fibrilación auricular y síndrome coronario agudo. 2019 Mar 20 [consulta 2019 Abr 13]. En: Cardioteca.com. Actualidad y Formación Cardiovascular. Cardio Teca Blog [Internet]. Madrid: Médica Soluciones Innova SL. [Consulta: 13 de abril 2019]. Disponible en: https://www.cardioteca.com/arritmias-blog/fibrilacion-auricular-blog/fibrilacion-auricular-anticoagulacion/193-fibrilacion-auricular-apixaban/3349-estudio-augustus-acc-2019-apixa- ban-en-fibrilacion-auricular-y-sindrome-coronario-agudo.html

3. El ensayo AUGUSTUS ha demostrado resultados de seguridad favorables de apixabán frente a antagonistas de la vitamina K [Internet]. Farmanews; 2019 [consulta 2019 Abr 13]. Disponible en: https://www. farmanews.com/Notasprensa/13333/El_ensayo_Augustus_ha_demostrado_resultados_de_seguridad_fav

4. Baumgartner H, Falk V, Bax JJ, De Bonis M, Hamm C, Holm PJ, et al. 2017 ESC/EACTS guidelines for the management of valvular heart disease. Eur Heart J. 2017;38(36):2739-91. doi: 10.1093/eurheartj/ehx391

5. Thyregod HG, Steinbruchel DA, Ihlemann N, Nissen H, Kjeldsen BJ, Petursson P, et al. Transcatheter versus surgical aortic valve replacement in patients with severe aortic valve stenosis: one-year results from the all-comers NOTION randomized clinical trial. J Am Coll Cardiol. 2015; 65(20):2184-94.doi: 10.1016/j.jacc.2015.03.014

6. Thyregod HG, Ihlemann N, Jørgensen TH, Nissen H, Kjeldsen BJ, Petursson P, et al. Five-Year clinical and echocardiographic outcomes from the Nordic Aortic Valve Intervention (NOTION) randomized clinical trial in lower surgical risk patients. Circulation. 2019 Feb 1. doi: 10.1161/CIRCULATIONAHA.118.036606

7. Mack MJ, Leon MB, Thourani VH, Makkar R, Kodali SK, Russo M, et al. Transcatheter aortic-valve replacement with a balloon-expandable valve in low-risk patients. N Engl J Med. 2019;380(18): 16951705. doi: 10.1056/NEJMoa1814052

8. Hughes S. PARTNER 3: TAVR Success in Low-Risk Patients [Internet]. New York: Medscape;2019 [Consulta 2019 Abr 12]. Disponible en: https://www. medscape.com/viewarticle/910503

9. Sociedad Española de Cardiología. La SEC te lleva a ACC19 [POST] [Internet]. Madrid: Sociedad Española de Cardiología;2019 [2019 Abr 15]. Disponible en: https:/www.youtube.com/watch?v= SSUT $\mathrm{xULKgkY \& t}=912 \mathrm{~s}$

10. Ibanez B, James S, Agewall S, Antunes MJ, Bucciarelli-Ducci C, Bueno H, et al. 2017 ESC Guidelines for the management of acute myocardial infarction in patients presenting with ST-segment elevation: the task force for the management of acute myocardial infarction in patients presenting with ST-segment elevation of the European Society of Cardiology (ESC). Eur Heart J. 2018;39(2):119-77. doi: 10.1093/eurheartj/ehx393

11. Neumann FJ, Sousa-Uva M, Ahlsson A, Alfonso F, Banning AP, Benedetto U, et al. 2018 ESC/EACTS Guidelines on myocardial revascularization. Eur Heart J. 2019; 40(2): 87-165. doi: 10.1093/eurheartj/ehy394 
12. Le May MR. Femoral Versus Radial Access for Primary PCI (SAFARI-STEMI) [Internet]. Rockville: U.S. National Library of Medicine; 2019 [Consulta 2019 Abr 7]. Disponible en: https://clinicaltrials. gov/ct2/show/NCT01398254

13. Bavry AA. SAfety and Efficacy of Femoral Access vs Radial Access in STEMI: SAFARI-STEMI [Internet]. Washington DC: American College of Cardiology; 2019 [Consulta 2019 Abr 7]. Disponible en: https://www. acc.org/latest-in-cardiology/clinical-trials/2019/03/16/ 23/57/safari-stemi

14. Le May M. SAFARI-STEMI: Similar 30-day mortality for radial vs. femoral access PCI [Internet]. Washington DC: American College of Cardiology; 2019 [Consulta 2019 Abr 7 ]. Disponible en: https://www. acc.org/latest-in-cardiology/articles/2019/03/08/15/32/ mon-1045am-safari-stemi-femoral-access-vs-radialaccess-for-primary-pci-acc-2019

15. Al Asnag M. ACC 2019 Day 3: SAFARI-STEMI, STOPDAPT-2, SMART-CHOICE and more. Reported from the ACC 2019 (ACC.19) scientific sessions in New Orleans [Internet]. PCR Online; 2019 [Consulta 2019 Abr 7]. Disponible en: https://www.pcronline.com/News/Whats-new-on-PCRonline/2019/ACC2019-Day-3-SAFARI-STEMI.-STOPDAPT-2.-SMA RT-CHOICE

16. O'Riordan M [Internet]. Marzo 18, 2019. SAFARI Surprise: No Survival or Bleeding Advantage With Radial Over Femoral Access in STEMI. [Consulta: 7 de abril 2019]. Disponible en: https://www.tctmd. com/news/safari-surprise-no-survival-or-bleeding-advantage-radial-over-femoral-access-stemi

17. Domei T, Morimoto T, Shiomi H, Natsuaki M, Totota T, Takagi K, et al. One-month dual antiplatelet therapy followed by clopidogrel monotherapy vs. standard 12-month dual antiplatelet therapy with clopidogrel after drug-eluting stent ImplantationSTOPDAPT-2 [Internet]. 2019 [Consulta 2019 Abr 6].Disponible en: https://www.acc.org/ / media/Clinical/PDF-Files/Approved-PDFs/2019/03/15/
ACC19_Slides/Mar18_Mon/1145amET_STOPDAPT2-acc-2019.pdf

18. Wermers J. Short DAPT at least non-inferior to standard DAPT, 2 studies show [Internet]. CRTonline; 2019 [Consulta 2019 Abr 8].Disponible en: http://www.crtonline.org/news-detail/short-daptat-least-non-inferior-to-standard-dapt-

19. Maxwell YL. Dropping Aspirin: two trials explore P2Y12 monotherapy after short-term DAPT Post-PCI. TCTMD; 2019 [Consulta 2019 Abr 8]. Disponible en: https://www.tctmd.com/news/droppingaspirin-two-trials-explore-p2y12-monotherapy-after- short-term-dapt-post-pci

20. Turakhia MP, Desai M, Hedlin H, Rajmane A, Talati N, Ferris T, et al. Rationale and design of a large-scale, app-based study to identify cardiac arrhythmias using a smartwatch: the apple heart study. Am Heart J. 2019; 207:66-75. doi: 10.1016/ j.ahj.2018.09.002.

21. Turakhia MP, Pérez M. Results of a large-scale, app-based study to identify cardiac arrhythmias using a smartwatch: the Apple Heart Study [Internet]. Stanford: California; 2019 [Consulta 2019 Abr 1]. Disponible en: https://www.acc.org/ /media/Clinical/PDF-Files/Approved-PDFs/2019/03/15/ ACC19_Slides/Mar16_Sat/10amET_ Apple-Heart-Study-acc-2019.pdf

22. Apple heart study identifies AFib in small group of apple watch wearers [Internet]. Washington: American College of Cardiology; 2019 [Consulta 2019 Abr 1]. Disponible en: https://www.acc.org/latest-in-cardiology/articles/2019/03/08/15/32/sat-9am-appleheart-study-acc-2019

23. Apple heart study gives glimpse into how wearable technology may help flag heart rhythm problems [Internet]. Washington: American College of Cardiology; 2019 [Consulta 2019 Apr 1]. Disponible en: https://www.acc.org/about-acc/press-releases/2019/ 03/16/10/52/apple-heart-study-gives-glimpseinto-how-wearable-technology-may-helpflag-heart-rhythm-problems 